\title{
Evaluation of lymph node metastases in gastric cancer using magnetic resonance imaging with ultrasmall superparamagnetic iron oxide (USPIO): diagnostic performance in post-contrast images using new diagnostic criteria
}

\author{
Takaya Tokuhara ${ }^{1}$, Nobuhiko Tanigawa ${ }^{1}$, Mitsuru Matsuki $^{2}$, Eiji Nomura ${ }^{1}$, Hideaki Mabuchi $^{1}$, \\ Sang-Woong Lee $^{1}$, Yoshiaki Tatsumi ${ }^{1}$, Haruto Nishimura ${ }^{1}$, Ryoji Yoshinaka ${ }^{1}$, Yoshitaka Kurisu ${ }^{3}$, \\ and ISAMU NARABAYASHI ${ }^{2}$ \\ ${ }^{1}$ Department of General and Gastroenterological Surgery, Osaka Medical College, 2-7 Daigaku-machi, Takatsuki, Osaka 569-8686, Japan \\ ${ }^{2}$ Department of Radiology, Osaka Medical College, Osaka, Japan \\ ${ }^{3}$ Department of Pathology, Osaka Medical College, Osaka, Japan
}

\begin{abstract}
Background. We assessed the value of magnetic resonance imaging (MRI), using ultrasmall superparamagnetic iron oxide (USPIO) with new diagnostic criteria, in the evaluation of regional lymph node metastases in gastric cancer.

Methods. Thirty-one patients with gastric cancer were enrolled. 1000 lymph nodes were dissected during surgery, and of these, 519 nodes $(51.9 \%)$ were identified by currently used MRI imaging analysis. We evaluated lymph nodes on USPIO-post-contrast T2*-weighted images using the following two criteria: (1) we diagnosed the nodes on $\mathrm{T} 2 *$-weighted images according to conventional criteria, where a node having an overall low signal intensity (pattern A) was nonmetastatic, while a node having partial (pattern B) or overall (pattern C) high signal intensity was metastatic; (2) we subdivided pattern $B$ nodes on T1-weighted images using the new criteria, in which a node for which the high-intensity area on T2*-weighted images was not defined as adipose tissue on T1-weighted images (pattern B1) was metastatic, while a node for which the high-intensity area was defined as adipose tissue (pattern B2) was nonmetastatic.

Results. (1) The results using the conventional criteria were 96.2\% sensitivity, $92.5 \%$ specificity, $76.3 \%$ positive predictive value (PPV), $\mathbf{9 9 . 0} \%$ negative predictive value (NPV), and 93.3\% accuracy. (2) The results using the new criteria were 96.2\% sensitivity, 98.3\% specificity, 90.1\% PPV, 99.0\% NPV, and $97.1 \%$ accuracy.

Conclusion. The assessment of lymph node metastases from USPIO-post-contrast MRI alone using the new criteria was useful in the diagnosis of regional lymph node metastases in gastric cancer.
\end{abstract}

Key words Ultrasmall superparamagnetic iron oxide (USPIO) · Ferumoxtran-10 · MRI · Lymph node metastasis · Gastric cancer

Offprint requests to: $\mathrm{N}$. Tanigawa

Received: March 3, 2008 / Accepted: August 23, 2008

\section{Introduction}

At present, various treatment methods are applied for patients with gastric cancer, including endoscopic treatment, open surgery, laparoscopic surgery, and neoadjuvant chemotherapy. Thus, an accurate preoperative diagnosis of regional lymph node metastases in gastric cancer is essential in deciding upon a treatment strategy. The guidelines for gastric cancer treatment published by the Japanese Gastric Cancer Association (JGCA) in both the first edition (2001) [1] and the second edition (2004) [2] recommend the mode of treatment based on the tumor stage according to evidencebased findings. Endoscopic treatment, like endoscopic mucosal resection (EMR) or endoscopic submucosal dissection (ESD), is recommended for lymph nodenegative patients, and partial and laparoscopic gastrectomy with limited extent lymph node dissection is recommended for patients who are possibly nodepositive. In patients with locally advanced cancer, neoadjuvant chemotherapy may sometimes be recommended for node-positive patients. Most recent clinical trials have demonstrated that paraaortic lymph node dissection does not lead to any survival benefit compared with the standard regional dissection, D2 [3]. Thus, precise lymph-node staging greatly influences the decision on the mode of treatment. For the evaluation of regional lymph nodes in gastric cancer, the nodes have generally been defined according to size or morphologic criteria in several imaging modalities, such as computed tomography (CT), endoscopic ultrasonography (EUS), and conventional magnetic resonance imaging (MRI), but these methods have not been confirmed as sufficiently effective [4-13]. Recently, the use of ultrasmall superparamagnetic iron oxide (USPIO), which is a lympho- 
graphic contrast medium for MR imaging, has been of interest in the evaluation of lymph nodes in patients with neck, prostatic, rectal, esophageal, breast, and gastric cancers [14-20].

We previously reported on the efficacy of MRI examination using USPIO with a comparison of preand post-contrast $\mathrm{T} 2 *$-weighted images in patients with gastric cancer, wherein the results demonstrated a diagnostic performance superior to those obtained by other diagnostic methods [20]. However, the following three problems arose in this examination: (1) the detectability of perigastric lymph nodes in reference to CT images was very low $(9.7 \%)$; (2) the performance of a comparison of $\mathrm{T} 2 *$-weighted images before and after administration of USPIO could affect patient compliance; and (3) the positive predictive value for $\mathrm{T} 2 *$-weighted images was relatively low, $85.5 \%$.

Accordingly, we carried out the present study to demonstrate the superiority of the introduction of T1- ,T2-, and $\mathrm{T} 2 *$-weighted images following the administration of USPIO, rather than the use of $\mathrm{T} 2 *$-weighted images before and after administration of USPIO (as reported in our previous work [20]), in the detection and accurate diagnosis of lymph node status in patients with gastric cancer.

\section{Patients, materials, and methods}

\section{Patients}

From June 2005 to June 2007, 31 patients (29 men and 2 women, ages ranging from 51 to 84 years; mean age, 67.8 years) were enrolled in the study. All patients were diagnosed with gastric cancer histologically via gastroendoscopy and were scheduled for surgery. All patients underwent primary tumor resection and regional lymph node dissection. This study protocol was approved by the ethics committee at Osaka Medical College. Written informed consent was obtained from all patients prior to the study. Of the 31 patients, 20 patients underwent distal gastrectomy, 1 patient underwent proximal gastrectomy, and 10 patients underwent total gastrectomy together with regional lymph node dissection. The definition of the anatomical locations of the regional lymph nodes was based on the Japanese classification of gastric carcinoma (JCGC) [21].

\section{Contrast agent}

Ultrasmall superparamagnetic iron oxide (USPIO; Ferumoxtran-10; Combidex; Advanced Magnetics, Boston, MA, USA) was reconstituted and diluted in $100 \mathrm{ml}$ of $0.9 \%$ normal saline, and administered intravenously through a $5.0-\mu \mathrm{m}$ microfilter by drip infusion for $60 \mathrm{~min}$, at a dose of $2.6 \mathrm{mg}$ of iron per kilogram of body weight. This dose was chosen on the basis of a phase II dose-ranging study and phase III results $[22,23]$. Adverse events were recorded from the time of administration of USPIO to surgery. In the 31 patients, no subjective or objective adverse events of any kind were experienced.

\section{MR imaging techniques}

Patients were scanned using a 1.5-T MRI scanner (SIGNA Horizon EchoSpeed LX 1.5TCV/I; General Electric Medical Systems, Milwaukee, WI, USA) in the supine position. Before MRI examination, patients were fasted for $3 \mathrm{~h}$. Glucagon (1 mg; Glucagon G Novo; Novonordisk, Tokyo, Japan) was administered intramuscularly approximately 5 min before the MRI examination unless it was contraindicated for the patient's condition.

Twenty-four hours after the administration of USPIO, T1-weighted, T2-weighted, and $\mathrm{T} 2 *$-weighted MR images were obtained. The parameters for the T1weighted MR images were as follows: repetition time/ echo time, $34 / 8.0 \mathrm{~ms}$; flip angle, $80^{\circ}$; echo train length, 1 ; section thickness, $5 \mathrm{~mm}$; field of view, $32 \times 24 \mathrm{~cm}$; matrix, $512 \times 192$; number of signals acquired, 1 . The parameters for the T2-weighted MR images were as follows: repetition time/echo time, $2000 / 97.6 \mathrm{~ms}$; flip angle, $90^{\circ}$; echo train length, 1 ; section thickness, $5 \mathrm{~mm}$; field of view, $32 \times 24 \mathrm{~cm}$; matrix, $512 \times 192$; number of signals acquired, 1 .The parameters for the $\mathrm{T} 2 *$-weighted MR images were as follows: fast spoiled gradient-echo (GRE), repetition time/echo time, 135/7.6 ms; flip angle, $60^{\circ}$; echo train length, 1 ; section thickness, $5 \mathrm{~mm}$; field of view, $32 \times 24 \mathrm{~cm}$; matrix, $512 \times 192$; number of signals acquired, 1 . Surgery was performed from 1 to 6 days (mean, 1.9 days) after MRI examination.

\section{Image analysis}

Before surgery, all images were analyzed independently by one radiologist (M. M.) and one surgeon (T. T.) who were well trained in MRI. In cases of discrepancy between the two readers, a consensus was reached through discussion. We detected regional lymph nodes on post-contrast T1- and T2-weighted images.

(1) We evaluated lymph nodes on post-contrast T2*weighted images and diagnosed lymph nodes using the following previously reported criteria: a node with pattern A having an overall low signal intensity due to diffuse uptake of USPIO was diagnosed as nonmetastatic; while a node with pattern B having a partial high signal intensity due to partial uptake of USPIO, or a node with pattern $\mathrm{C}$ having an 

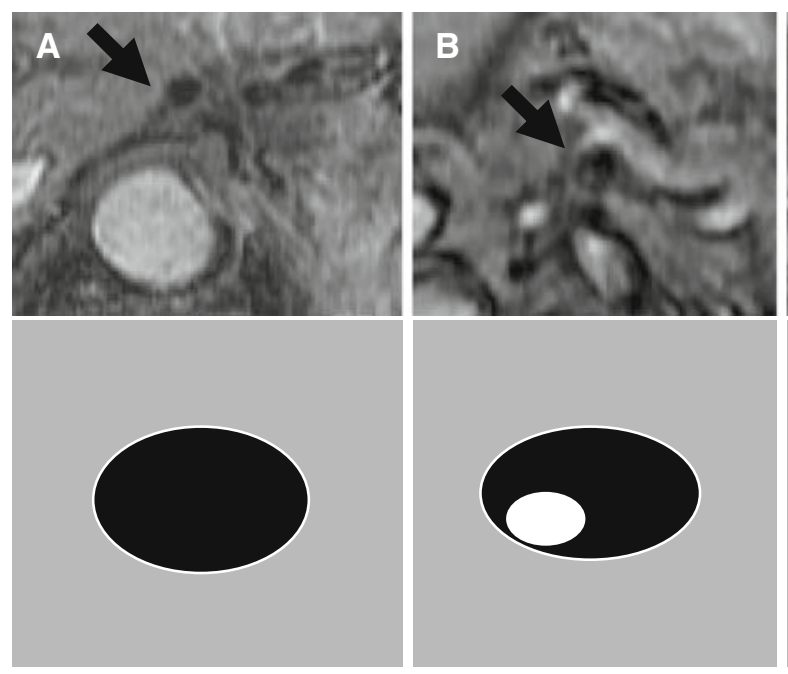

Fig. 1A-C. Conventional classification of lymph nodes on ultrasmall superparamagnetic iron oxide (USPIO) postcontrast T2*-weighted images. A Pattern A; 68-year-old man (gastric cancer on the lesser curvature of lower body, T2N2M0, stage II). The $\mathrm{T} 2 *$-weighted image shows a lymph node with an overall low signal intensity (pattern A) along the left gastric artery (no. 7; arrow), which was histopathologically nonmetastatic. B Pattern B; 79-year-old man (gastric cancer on the lesser curvature of the antrum, T3N2M0, stage IIIB). The

overall high signal intensity due to no uptake of USPIO, was diagnosed as metastatic (Fig. 1).

(2) We evaluated lymph nodes on both post-contrast T2*- and T1-weighted images. Here, we subdivided the nodes with pattern $\mathrm{B}$, referring to T1-weighted images as follows: a node for which the highintensity area on $\mathrm{T} 2{ }^{*}$-weighted images was not defined as adipose tissue on T1-weighted images (pattern B1) was metastatic (Fig. 2), while a node for which the high-intensity area was defined as adipose tissue (pattern B2) was nonmetastatic (Fig. 3).

\section{Histopathological evaluation}

To achieve the most precise correlation between MRI lymph node imagings and histopathological findings, histopathological examination was performed in lymph nodes $5 \mathrm{~mm}$ or more in size [20]. The surgeons operated with no information on the MRI images. The dissected lymph nodes were fixed in formalin for at least $24 \mathrm{~h}$. The largest cut surface of each lymph node was sectioned and stained with hematoxylin and eosin. A pathologist (Y. K.) diagnosed each lymph node as metastatic or nonmetastatic. One surgeon (T. T.) and one pathologist (Y. K.) performed a node-to-node correlation between the post-contrast MRI and histopathological findings.
T2*-weighted image shows a lymph node with a partial high signal intensity (pattern B) along the left gastric artery (no. 7; arrow), which was histopathologically metastatic. C Pattern C; 72-year-old man (gastric cancer on the anterior wall of the middle body, T2N1M0, stage II). The T2*-weighted image shows a perigastric lymph node (no. 3; arrow) with an overall high signal intensity (pattern $\mathrm{C}$ ), which was histopathologically metastatic

\section{Statistical analysis}

We calculated sensitivity, specificity, positive and negative predictive values, and overall accuracy based on the lymph nodes using the diagnostic criteria that refer to patterns $\mathrm{A}, \mathrm{B}$, and $\mathrm{C}$ and patterns $\mathrm{A}, \mathrm{B} 1, \mathrm{~B} 2$, and $\mathrm{C}$, individually in the total node region, in the perigastric node region, in the retroperitoneal lymph node region, and in the paraaortic lymph node region.

\section{Results}

(1) According to the JCGC, 2 patients had stage IA disease; 3 , stage IB; 3 , stage II; 6 , stage IIIA; 7, stage IIIB; and 10, stage IV, while based on the TNM classification, 3 had stage IA disease; 2 , stage IB; 5 , stage II; 7, stage IIIA; and 14, stage IV [24].

In terms of tumor histology, 14 tumors were tubular adenocarcinomas (well-differentiated type), 5 were tubular adenocarcinomas (moderately differentiated type), 5 were solid-type poorly differentiated adenocarcinomas, 6 were nonsolid-type poorly differentiated adenocarcinomas, and 1 was a mucinous adenocarcinoma.

(2) A total of 1000 lymph nodes from the 31 patients, including 184 histologically proven metastatic lymph nodes, were dissected during surgery. As 

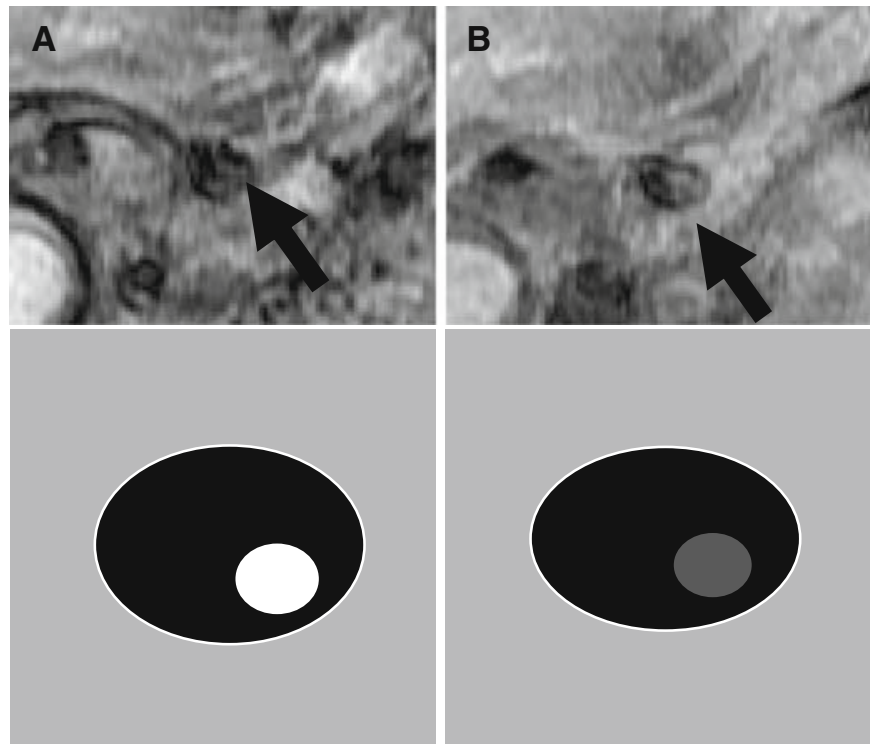

Fig. 2A-C. Pattern B1 based on the new classification of lymph nodes on USPIO post-contrast $\mathrm{T} 2 *$ and T1-weighted images: 76-year-old man (gastric cancer on the lesser curvature of the cardia, T2N1M0, stage II). A The T2*-weighted image shows a perigastric lymph node (no. 1; arrow) with a partial high signal intensity (pattern B). B The T1-weighted

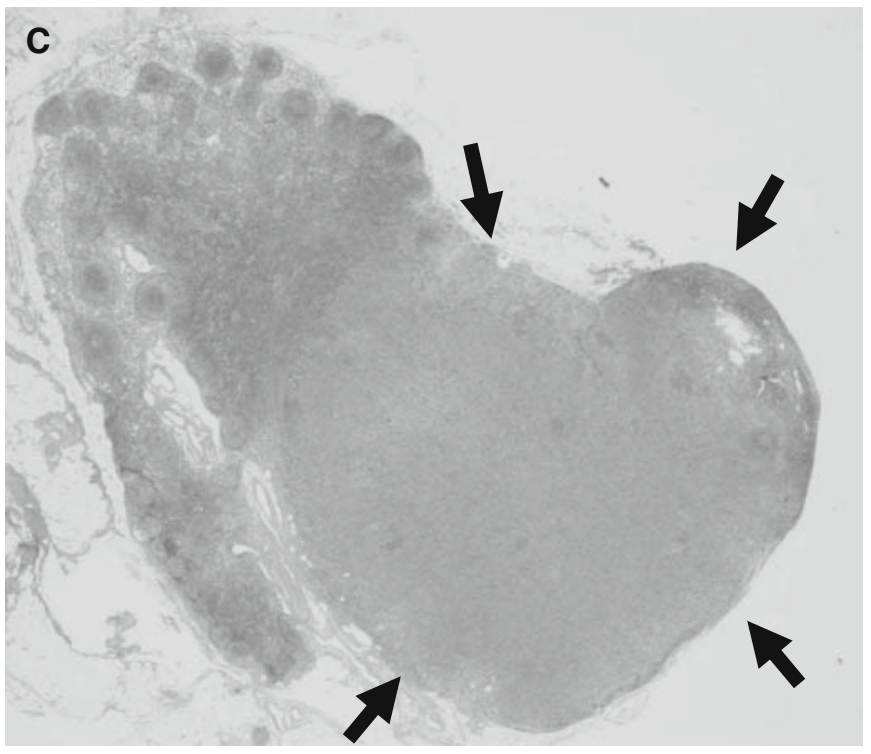

image shows that the partial high-intensity area on the $\mathrm{T} 2 *$ weighted image (arrow) was not defined as adipose tissue (pattern B1). C Histopathological specimen demonstrates a metastatic lymph node. The partial high-intensity area on the $\mathrm{T} 2 *$-weighted image is histopathologically identical to the metastatic focus (arrows). $\mathrm{H} \& \mathrm{E}$
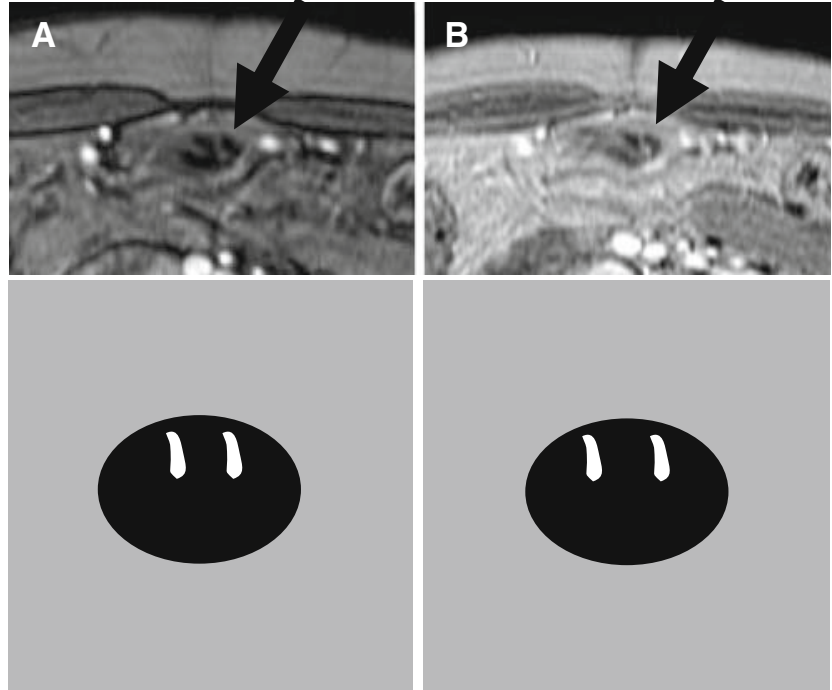

Fig. 3A-C. Pattern B2 based on the new classification of lymph nodes on USPIO post-contrast T2* and T1-weighted images; 52-year-old man (gastric cancer on the greater curvature of lower body, T1N0M0, stage IA). A The T2*-weighted image shows a perigastric lymph node (no. 4d; arrow) with a partial high signal intensity (pattern B). B The T1-weighted

for lymph node regions, 661 perigastric lymph nodes (152 metastatic and 509 nonmetastatic nodes), 325 retroperitoneal lymph nodes (32 metastatic and 293 nonmetastatic nodes), and 14 paraaortic lymph nodes (14 nonmetastatic nodes) were dissected.

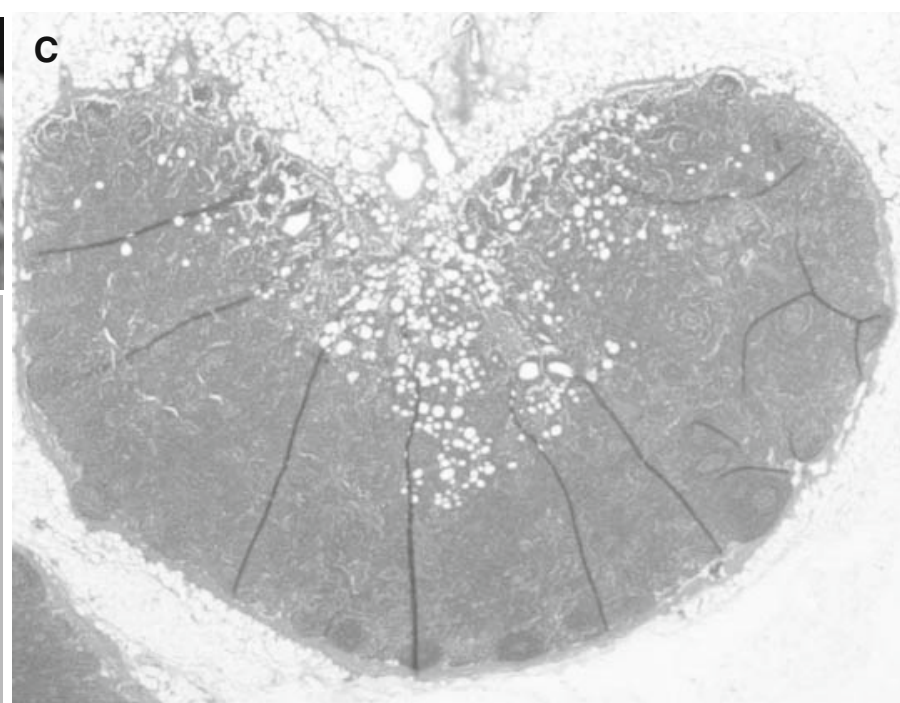

image shows that the partial high-intensity area on the $\mathrm{T} 2 *_{\text {- }}$ weighted image (arrow) was defined as adipose tissue (pattern B2). C Histopathological specimen demonstrates a nonmetastatic lymph node. The partial high-intensity area on the T2*weighted image is histopathologically identical to the adipose tissue in the lymphatic hilum. H\&E

(3) In the total node region, 519 of 1000 nodes (51.9\%) were identified by the currently used MRI imaging analysis. Of these, 104 lymph nodes $(20.0 \%)$ were metastatic. In the perigastric node region, 311 of 661 perigastric nodes $(47.0 \%)$ were identified. Of these, 80 nodes $(25.7 \%)$ were metastatic. In the 
Table 1. Distribution of lymph nodes based on the conventional diagnostic criteria

\begin{tabular}{lcc}
\hline Pattern & Metastatic nodes & Nonmetastatic nodes \\
\hline A & 4 & 384 \\
B & 8 & 28 \\
C & 92 & 3 \\
\hline
\end{tabular}

Table 2. Lymph-node-based results using the conventional and new diagnostic criteria

\begin{tabular}{lcc}
\hline & $\begin{array}{c}\text { Conventional } \\
\text { diagnostic criteria }\end{array}$ & $\begin{array}{c}\text { New } \\
\text { diagnostic criteria }\end{array}$ \\
\hline Sensitivity & $96.2 \%(100 / 104)$ & $96.2 \%(100 / 104)$ \\
Specificity & $92.5 \%(384 / 415)$ & $98.3 \%(404 / 411)$ \\
PPV & $76.3 \%(100 / 131)$ & $90.1 \%(100 / 111)$ \\
NPV & $99.0 \%(384 / 388)$ & $99.0 \%(404 / 408)$ \\
Accuracy & $93.3 \%(484 / 519)$ & $97.1 \%(504 / 519)$ \\
\hline
\end{tabular}

Numbers in parentheses are numbers of lymph nodes

PPV, positive predictive value; NPV, negative predictive value

retroperitoneal node region, 198 of 325 nodes $(60.9 \%)$ were identified. Of these, 24 nodes $(12.1 \%)$ were metastatic. In the paraaortic node region, 10 $(71.4 \%)$ of 14 paraaortic nodes were identified. Of these, all nodes were nonmetastatic.

(4) In the 519 identified nodes, there were 384 histopathologically nonmetastatic $(74.0 \%)$ and 4 metastatic nodes $(0.8 \%)$ among 388 nodes $(74.8 \%)$ with pattern A. Among 36 nodes $(6.9 \%)$ with pattern B, $28(5.4 \%)$ were histopathologically nonmetastatic and 8 nodes $(1.5 \%)$ were metastatic. There were $3(0.6 \%)$ histopathologically nonmetastatic and 92 nodes $(17.7 \%)$ that were metastatic among the 95 nodes $(18.3 \%)$ with pattern C (Table 1). Based on the conventional diagnostic criteria, these results exhibited $96.2 \%$ sensitivity, $92.5 \%$ specificity, $76.3 \%$ positive predictive value (PPV), 99.0\% negative predictive value (NPV), and $93.3 \%$ accuracy (Table 2 ). According to the new criteria, with the subdivision of pattern $\mathrm{B}$, the 36 pattern $\mathrm{B}$ lymph nodes $(6.9 \%)$ were divided into 16 pattern B1 nodes and 20 pattern B2 nodes. Of these 16 nodes with pattern B1 $(6.9 \%), 8$ were histopathologically nonmetastatic and 8 nodes were metastatic. All of the 20 nodes with pattern B2 (3.9\%) were histopathologically nonmetastatic (Table 3). Based on the new diagnostic criteria, the results exhibited $96.2 \%$ sensitivity, 98.3\% specificity, 90.1\% PPV, 99.0\% NPV, and $97.1 \%$ accuracy (Table 2 ).

(5) In the 311 perigastric nodes identified with postcontrast MRI, the results exhibited $100 \%$ sensitivity, $97.4 \%$ specificity, $93.0 \% \mathrm{PPV}, 100 \% \mathrm{NPV}$, and $98.1 \%$ accuracy using the new diagnostic criteria.
Table 3. Distribution of pattern B lymph nodes based on the new diagnostic criteria

\begin{tabular}{lcc}
\hline Pattern & Metastatic nodes & Nonmetastatic nodes \\
\hline B1 & 8 & 8 \\
B2 & 0 & 20 \\
\hline
\end{tabular}

(6) In the 198 retroperitoneal nodes identified with post-contrast MRI, the results exhibited $83.3 \%$ sensitivity, 97.1\% specificity, 80.0\% PPV, 98.1\% NPV, and $95.5 \%$ accuracy with the new diagnostic criteria. The reason that the results for retroperitoneal lymph nodes were worse than those for other locations is unclear, but may be related to an insufficient number being tested.

(7) In the ten paraaortic nodes identified with postcontrast MRI, the results exhibited $100 \%$ specificity, $100 \% \mathrm{NPV}$, and $100 \%$ accuracy with the new diagnostic criteria. According to these results, it seems that this new method for evaluating lymph node status may have the potential to correctly identify the subgroup of patients for whom palliative treatment is indicated or patients who would need D3 lymphadenectomy.

\section{Discussion}

It has been reported that lymph node metastasis is an important prognostic factor in gastric cancer [25, 26]. And so the treatment strategies for gastric cancer are diverse as to $\mathrm{N}$-staging, including endoscopic treatment, laparoscopic surgery, open surgery, and neoadjuvant chemotherapy, as indicated in the guidelines for gastric cancer treatment [1,2]. Endoscopic treatment can be performed for some intramucosal and submucosal cancers with no lymph node metastases [27]. However, laparoscopic surgery cannot be used for patients with retroperitoneal lymph node metastases [28]. The utility of neoadjuvant chemotherapy has recently been discussed for some patients with advanced gastric cancer with suspected extensive lymph node metastases $[29,30]$. Therefore, preoperative assessment of regional lymph node involvement in gastric cancer is essential in deciding upon a treatment strategy.

In the evaluation of regional lymph nodes in gastric cancer, various imaging modalities, such as computed tomography (CT), endoscopic ultrasonography (EUS), and conventional magnetic resonance imaging (MRI) have been used, with lymph nodes assessed according to size or morphologic criteria. The reported accuracy of CT, EUS, and conventional MRI for the N-staging of gastric cancer was $43 \%-80 \%, 65 \%-87 \%$, and $34 \%-$ $65 \%$, respectively, and this level of diagnostic perfor- 
mance was not sufficient for making a decision among treatment strategies for gastric cancer [4-13]. Recently, a lymphographic contrast medium of iron-containing nanoparticles, referred to as ultrasmall superparamagnetic iron oxide (USPIO), has received attention in the evaluation of lymph nodes in various types of malignancy, such as neck, prostatic, rectal esophageal, breast, and gastric cancers [14-20]. After the intravenous injection of USPIO, the nanoparticles circulate systemically and accumulate in the interstitium. These nanoparticles in the interstitium are internalized by macrophages and are drained through lymphatic vessels to the lymph nodes. The iron-containing nanoparticles accumulate in normal lymph nodes, which enhances susceptibility effects and reduces the nodal $\mathrm{T}_{2}^{*}$ signal. In contrast, because metastatic lymph nodes lack reticuloendothelial activity, they are unable to take up the iron-containing nanoparticles that cause local field heterogeneity [22].

We have previously reported on the efficacy of MRI examination using USPIO in the evaluation of lymph nodes in patients with gastric cancer, wherein the results demonstrated that the sensitivity, specificity, PPV, NPV and accuracy were $100 \%, 92.6 \%, 85.5 \%, 100 \%$, and $94.8 \%$, respectively. These parameters were far superior to those achieved previously with evaluations using other diagnostic methods such as CT, US, and conventional MRI. However, there were several problems, as follows.

(1) In our previous study, lymph nodes were identified on pre- and post-contrast $\mathrm{T} 2 *$-weighted images in reference to CT images, and the detectability of perigastric lymph nodes was relatively low $(9.7 \%)$. The difficulty in the detection of lymph nodes by this technique was considered to be due to the fact that the morphology and degree of distension of the stomach changed between the pre- and post-contrast $\mathrm{T} 2 *$-weighted images, and this occurred likewise for the CT images, which were performed on different days. Harisinghani et al. [31] emphasized that, in comparison to $\mathrm{T} 2 *$-weighted images, T1and T2-weighted images were more suitable for the identification of lymph nodes because of the high spatial resolution. Therefore, we selected the method in which we detected lymph nodes on postcontrast T1- and T2-weighted images and evaluated them on post-contrast $\mathrm{T} 2 *$-weighted images. As a result, the detectability of perigastric lymph nodes increased to $47.0 \%$.

(2) It was reported that there was no significant difference in diagnostic performance between a combined study of pre- and post-contrast images and a post-contrast study alone [19, 31]. Therefore, we tried, in this present study, to examine the post- contrast study alone, and compared the results to those from our previous study. The diagnostic performance in the post-contrast study alone gave sensitivity, specificity, and accuracy of $96.2 \%, 92.5 \%$, and $93.3 \%$, respectively, values which were rather similar to those from our previous study, where the sensitivity, specificity, and accuracy were $100 \%$, $92.6 \%$, and $94.8 \%$, respectively.

(3) Recently, it was reported that a partial high signal in the lymph nodes on post-contrast $\mathrm{T} 2 *$-weighted images could reflect tumor replacement or adipose tissue in the lymphatic hilum, and, therefore, careful scrutiny of T1-weighted images was required to identify a fatty central hilum [18]. We then utilized the new diagnostic criteria with subdivision of the pattern B nodes referring to T1-weighted images as follows: nodes with a high intensity area on $\mathrm{T} 2{ }^{*}$ weighted images, which was not defined as adipose tissue on the T1-weighted images (pattern B1), were diagnosed as metastatic. At the same time, the nodes with a high intensity area on the $\mathrm{T} 2 *$-weighted images, which was defined as adipose tissue on T1weighted images (pattern B2), were diagnosed as nonmetastatic. As a result, the diagnostic performance with the new diagnostic criteria gave specificity and accuracy of $98.3 \%$, and $97.1 \%$, respectively, values which were superior to those from our previous study [20], where specificity and accuracy were $92.6 \%$, and $94.8 \%$, respectively.

\section{Conclusion}

The present study indicates that the assessment of lymph node metastases on USPIO-post-contrast MRI alone using the new diagnostic criteria was useful in the diagnosis of regional lymph node metastases in gastric cancer. This diagnostic performance can contribute to making better decisions on the appropriate treatment strategy in patients with gastric cancer.

Acknowledgments We are grateful to Paula Jacobs, Vice President of Advanced Magnetics, for providing the contrast agent.

\section{References}

1. Japanese Gastric Cancer Association. Gastric cancer treatment guidelines in Japan. 1st ed (in Japanese). Tokyo: Kanehara; 2001.

2. Japanese Gastric Cancer Association. Gastric cancer treatment guidelines in Japan. 2nd ed (in Japanese). Tokyo: Kanehara; 2004.

3. Sasako M, Sano T, Yamamoto S, Kurokawa Y, Nashimoto A, Kurita A, et al. D2 lymphadenectomy alone or with para-aortic nodal dissection for gastric cancer. N Engl J Med 2008;359:453-62. 
4. Grimm H, Soehendra N, Hapmper K, Maas R. Contribution of endoscopy to preoperative staging in esophageal and stomach cancer. Chirurg 1989;60:684-9.

5. Boku T, Nakane Y, Okumura S, Okamoto M, Okamura N, Kasamatsu S, et al. Preoperative diagnosis of gastric cancer using plain computed tomography and dynamic computed tomography (in Japanese). J Japan Soc Gastroenterol Surg 1991;24:757-62.

6. Nattermann C, Galbenu-Grünwald R, Nier H, Dancygier H. Endoscopic ultrasound in TN staging of stomach cancer. A comparison with computerized tomography and conventional ultrasound. Z Gesamte Inn Med 1993;48:60-4.

7. Peng DS, Jan CM, Wang WM, Chen LT, Su YC, Liu GC, et al. Computed tomography, endoscopic ultrasonography and intraoperative assessment in TN staging of gastric carcinoma. J Formos Med Assoc 1996;95:378-85.

8. Kim AY, Han JK, Seong CK, Kim TK, Choi BI. MRI in staging advanced gastric cancer: is it useful compared with spiral CT? J Comput Assist Tomogr 2000;24:389-94.

9. Sohn KM, Lee JM, Lee SY, Ahn BY, Park SM, Kim KM. Comparing MR imaging and $\mathrm{CT}$ in the staging of gastric carcinoma. AJR Am J Roentgenol 2000;174:1551-7.

10. Polkowski M, Palucki J, Wronska E, Szawlowski A, Nasierowska-Guttmejer A, Butruk E. Endosonography versus helical computed tomography for locoregional staging of gastric cancer. Endoscopy 2004:36:617-23.

11. Bhandari S, Shim CS, Kim JH, Jung IS, Cho JY, Lee JS, et al. Usefulness of three-dimensional, multidetector row CT (virtual gastroscopy and multiplanar reconstruction) in the evaluation of gastric cancer: a comparison with conventional endoscopy, EUS, and histopathology. Gastrointest Endosc 2004;59:619-26.

12. Arocena MG, Barturen A, Bujanda L, Casado O, Ramírez MM, Oleagoitia JM, et al. MRI and endoscopic ultrasonography in the staging of gastric cancer. Rev Esp Enferm Dig 2006;98:582-90.

13. Chen CY, Hsu JS, Wu DC, Kang WY, Hsieh JS, Jaw TS, et al. Gastric cancer: preoperative local staging with 3D multi-detector row $\mathrm{CT}$ - correlation with surgical and histopathological results. Radiology 2007;242:472-82.

14. Anzai Y, Prince MR. Iron oxide-enhanced MR lymphography: the evaluation of cervical lymph node metastases in head and neck cancer. J Magn Reson Imaging 1997;7:75-81.

15. Mack MG, Balzer JO, Straub R, Eichler K, Vogl TJ. Superparamagnetic iron oxide-enhanced MR imaging of head and neck lymph nodes. Radiology 2002;222:239-44.

16. Harisinghani MG, Barentsz J, Hahn PF, Deserno WM, Tabatabaei S, van de $\mathrm{Kaa} \mathrm{CH}$, et al. Noninvasive detection of clinically occult lymph node metastases in prostate cancer. N Engl J Med 2003;348:2491-9.

17. Koh DM, Brown G, Temple L, Blake H, Raja A, Toomey P, et al. Rectal cancer: mesorectal lymph nodes at MR imaging with USPIO versus histopathologic findings - initial observations. Radiology 2004;231:91-9.

18. Nishimura H, Tanigawa N, Hiramatsu M, Tatsumi Y, Matsuki M, Narabayashi I. Preoperative esophageal cancer staging: magnetic resonance imaging of lymph node with ferumoxtran-10, an ultrasmall superparamagnetic iron oxide. J Am Coll Surg 2006; 202:604-11.

19. Harada T, Tanigawa N, Matsuki M, Nohara T, Narabayashi I. Evaluation of lymph node metastases of breast cancer using ultrasmall superparamagnetic iron oxide-enhanced magnetic resonance imaging. Eur J Radiol 2007;63:401-7.

20. Tasumi Y, Tanigawa N, Nishimura H, Nomura E, Mabuchi H, Matsuki M, et al. Preoperative diagnosis of lymph node metastasis in gastric cancer by magnetic resonance imaging with ferumoxtran-10. Gastric Cancer 2006;9:120-8.

21. Japanese Gastric Cancer Association. Japanese classification of gastric carcinoma - 2nd English edition - Gastric Cancer 1998;1:10-24

22. Hudgins PA, Anzai Y, Morris MR, Lucas MA. Ferumoxtran-10, a superparamagnetic iron oxide as a magnetic resonance enhancement agent for imaging lymph nodes: a phase 2 dose study. AJNR Am J Neuroradiol 2002;23:649-56.

23. Anzai Y, Piccoli CW, Outwater EK, Stanford W, Bluemke DA, Nurenberg P, et al. Evaluation of neck and body metastases to nodes with ferumoxtran 10-enhanced MR imaging: phase III safety and efficacy study. Radiology 2003;228:777-88.

24. Sobin LH, Wikkekind C. TNM classification of malignant tumors. 6th ed. New York: Wiley-Liss; 2002.

25. Yokota T, Ishiyama S, Saito T, Teshima S, Narushima Y, Murata $\mathrm{K}$, et al. Lymph node metastasis as a significant prognostic factor in gastric cancer: a multiple logistic regression analysis. Scand J Gastroenterol 2004:39:380-4.

26. Isozaki H, Fujii K, Nomura E, Mabuchi H, Nishiguchi K, Hara $\mathrm{K}$, et al. Prognostic factors of advanced gastric carcinoma without serosal invasion (pT2 gastric carcinoma). Hepatogastroenterology 1999;46:2669-72.

27. Gotoda T, Yanagisawa A, Sasako M, Ono H, Nakanishi Y, Shimoda $\mathrm{T}$, et al. Incidence of lymph node metastasis from early gastric cancer: estimation with large number of cases at two large centers. Gastric Cancer 2000;3:219-25.

28. Tanigawa N, Nomura E, Mabuchi H, Lee SW, Yoshinaka T. Laparoscopic (assisted) distal gastrectomy for cancer; from a positive viewpoint (in Japanese). Geka Chiryo 2007;69:630-6.

29. Schuhmacher CP, Fink U, Becker K, Busch R, Dittler HJ, Mueller $\mathrm{J}$, et al. Neoadjuvant therapy for patients with locally advanced gastric carcinoma with etoposide, doxorubicin, and cisplatin. Closing results after 5 years of follow-up. Cancer 2001;91: 918-27.

30. Yoshida I, Sakurai Y, Komori Y, Tonomura S, Masui T, Shoji M, et al. Successful downstaging by S-1-based chemotherapy followed by surgical resections for gastric carcinoma with extensive distant lymph node metastasis: report of two cases and a review of cases with surgical resection after downstaging by S-1-based chemotherapy. Hepatogastroenterology 2005;52:978-84.

31. Harisinghani MG, Saksena MA, Hahn PF, King B, Kim J, Torabi MT, et al. Ferumoxtran-10-enhanced MR lymphangiography: does contrast-enhanced imaging alone suffice for accurate lymph node characterization? AJR Am J Roentgenol 2006;186: $144-8$. 